\title{
Impact of Biosynthesized Silver Nanoparticles on Bacterial Growth
}

\author{
Jishnu Basu ${ }^{1}$ and Tiffany Grimes ${ }^{1}$ \\ ${ }^{1}$ Plano Senior High School, Plano, TX, USA
}

\begin{abstract}
$\underline{\text { ABSTRACT }}$
Cystic Fibrosis is a genetic disease which causes the production of viscous mucus in airways which limits airflow and creates the perfect conditions for bacterial growth. Unfortunately, deaths due to bacterial infections in Cystic Fibrosis patients have increased as bacterial strains have developed antibiotic resistance. Researchers have found that silver nanoparticles offer a solution to growing antibiotic resistance due to how no resistance has been developed to them in clinical trials. Current research is focusing on the bio-synthesis of silver nanoparticles which does not produce the harmful waste products seen with the industrial production of silver nanoparticles. However, there is a lack of comparative research concerning the effectiveness of silver nanoparticles produced by different microorganisms, which is what the researcher's work addressed. The researcher's work primarily focused on determining how effective silver nanoparticles produced by different bacterial species were at inhibiting bacterial growth. Through the collection of nanoparticles via extracellular synthesis, antimicrobial assays were conducted to determine the efficacy of silver nanoparticles produced by different microorganisms. The results indicated that silver nanoparticles produced by $B$. subtilis were the most effective in inhibiting bacterial growth. This provides a crucial as research in the field should increasingly focus on bacteria which utilize assimilatory nitrate reduction like $B$. subtilis because of the increased efficacy of silver nanoparticles produced by this method in inhibiting bacterial growth in aerobic conditions. Advances in this area could increase the efficiency of nanoparticle production and make it viable for industrial production.
\end{abstract}

\section{Introduction}

In Cystic Fibrosis, bacterial infections pose a huge health risk due to the optimal conditions for bacterial growth created by the stagnant mucus. In fact, about $85 \%$ of the deaths in Cystic Fibrosis occur because of lung infections with bacterial infections of Pseudomonas aeruginosa, Staphylococcus aureus, and Burkholderia cepacian common. Current scientific research has focused on silver nanoparticles as a novel solution to the problem of antibiotic resistant bacterial strains developing across the world. Silver nanoparticles due to their nature have potent bactericidal effects and limited resistance developed against them. These bactericidal effects include the rupturing of the cell wall, the denaturing of critical cellular proteins including ribosomes, and the excessive build-up of reactive oxygen species (i.e. hydrogen peroxide, superoxide anion, hydroxyl radical, etc.). All of these antibacterial mechanisms combined with the limited resistance developed against them make silver nanoparticles a very prominent solution to the current problem.

Throughout scientific literature related to silver nanoparticles, a common point of interest has been the novel method of producing nanoparticles via green synthesis which involves using natural elements and microorganisms to produce silver nanoparticles. This has several advantages over physical or chemical means of production in that it is much more cost-effective, eco-friendly as it does not require hazardous stabilizing agents, and can easily be scaled up for industrial level applications. The most recent works suggest the unique role bacteria can play in producing silver nanoparticles because of their intrinsic ability to reduce nitrate. By exposing bacterial species to silver nitrate solutions, 
researchers have been able to successfully create silver nanoparticles as the bacteria reduce nitrate with their enzymes via extracellular synthesis. Specifically, most bacterial species reduce nitrate with the help of NADH-dependent enzymes including nitrate reductase. However, current research has failed to observe any potential differences in the antibacterial properties of silver nanoparticles produced by different bacterial species via extracellular synthesis. It is this area in which this work is focused in the hope that with the results three key contributions to scientific knowledge in the area could be made: 1) the identification of the bacterial species which could provide more potent silver nanoparticles, 2) the identification of characteristics to cross-reference for more effective bacterial species in the production process, and 3) the increased efficiency of the synthesis process to provide for easier industrial operations.

\section{Experimental Procedures}

\section{Preparation of Silver Nanoparticles}

Preparation began upon delivery of the samples of E. coli and B. subtilis monocultures to the laboratory. In two separate conical flasks $100 \mathrm{~mL}$ of nutrient broth were created with approximately 8 grams of dry mix per liter of distilled water with one flask labeled as "E. coli Synthesized" and the other as "B. subtilis Synthesized". The nutrient broth mixtures were placed in an autoclave for sterilization. The inoculation process involved using a Bunsen burner to create a flame to sterilize the inoculation loops with in between the inoculation of the nutrient broth mixtures with their respective bacterial monocultures. After the inoculation process, the flasks were placed in an incubator at 37 degrees Celsius for 36 hours for appropriate bacterial growth (Figure 1). After incubation, the samples of nutrient broth were centrifuged at 5,000 RPM for ten minutes to isolate the supernatant. $50 \mathrm{~mL}$ of the supernatant from each flask were then collected into two respective $250 \mathrm{~mL}$ Erlenmeyer flasks in addition to $50 \mathrm{~mL}$ of $1 \mathrm{mM}$ silver-nitrate solution. This solution of supernatant and the silver nitrate for was incubated for five days at 40 degrees Celsius. After five days, the silver nanoparticles were formed and a noticeable color change within the Erlenmeyer flasks indicated this.

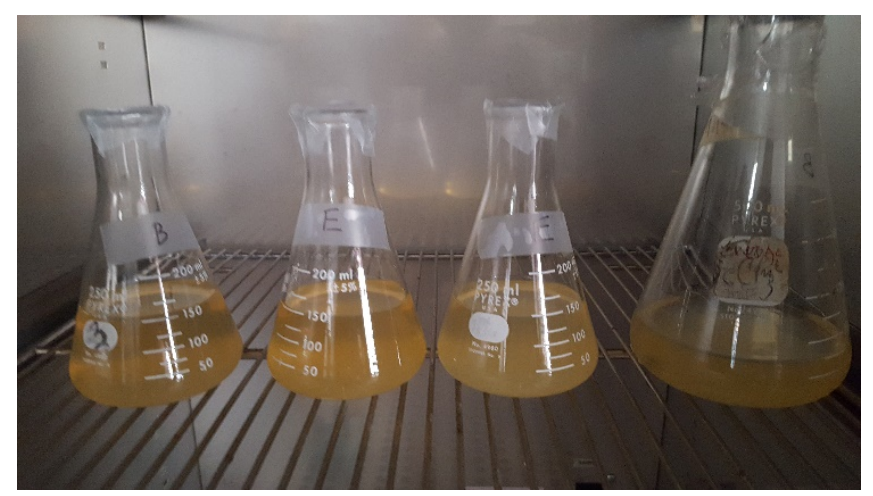

Figure 1. Images of silver nanoparticles during incubation process with color change indicative of formed silver nanoparticles.

\section{Agar Well Diffusion Assay}

Agar plates were prepared for the assay by heating approximately $300 \mathrm{~mL}$ of distilled water to the boiling point using a hot plate. In a $500 \mathrm{~mL}$ Erlenmeyer flask, 24 grams of nutrient agar per liter of heated distilled water were poured in. In small increments, the heated water from the Erlenmeyer flask was added to the nutrient agar with vigorous shaking after each increment. Afterwards, the mixture was placed in the autoclave to be properly sterilized. At this point, the liquid agar was poured into the labeled Petri dishes for the agar to set at room temperature. Once the agar was set, the 
same inoculation process as outlined above was used to introduce the bacterial monoculture of $P$. fluorescens on to the growth medium. The Petri dishes were placed in an incubator at 28 degrees Celsius for optimal bacterial growth for three days. After three days, 4 wells were created per Petri dish using a cork borer. In each well, 10 microliters of prepared silver nanoparticles were added using a micropipette. The control Petri Dish was left without any nanoparticles. After another 3 days, the zone of inhibition created by the diffusion of the silver nanoparticles into the agar was recorded.

\section{Statistical Analysis}

Statistical analysis of the data began with the calculation of the standard deviation and variance to get a better understanding of the spread of the data. The second set of analysis done was a one-tailed Student's t-test which tested the data to establish statistical significance and ensure the data was a result of only internal factors. The t-test had a critical value of $\mathrm{p}=0.05$ to establish statistical significance and was conducted through Microsoft Excel.

\section{Disposal}

A $10 \%$ bleach solution was poured into all liquid and solid growth medium with any microorganisms/bacterial content or growth for biohazard sterilization. Any remaining glassware or material were washed thoroughly with soap, water, and bleach before drying.

\section{Results}

\section{Agar Well Diffusion Assay Results}

The results of the agar well diffusion assay as measured by the zone of inhibition created by the silver nanoparticles were tabulated by averaging the results of each Petri dish. Each Petri Dish had four trials conducted on it (Table $1 \&$ Figure 2). Only two datapoints, one from Dish 1 of the B. subtilis trials and another from 3 of the $E$. coli trials had to be removed due to accidental spillage of the silver nanoparticles solution on that portion of the Petri Dish.

Table 1. Results from agar well diffusion assay displayed as measure of average zone of inhibition in $\mathrm{cm}$.

\begin{tabular}{|c|c|c|c|c|c|}
\hline \multicolumn{7}{|c|}{ Average Zone of Inhibition for Silver Nanoparticles in cm } \\
\hline & Dish 1 & Dish 2 & Dish 3 & Dish 4 & Average \\
\hline Control & 0 & 0 & 0 & 0 & 0 \\
\hline B. subtilis & 0.52 & 0.64 & 0.70 & 0.49 & 0.59 \\
\hline E. coli & 0.27 & 0.16 & 0.60 & 0.21 & 0.31 \\
\hline
\end{tabular}




\section{Average Zone of Inhibition for Silver Nanoparticles}

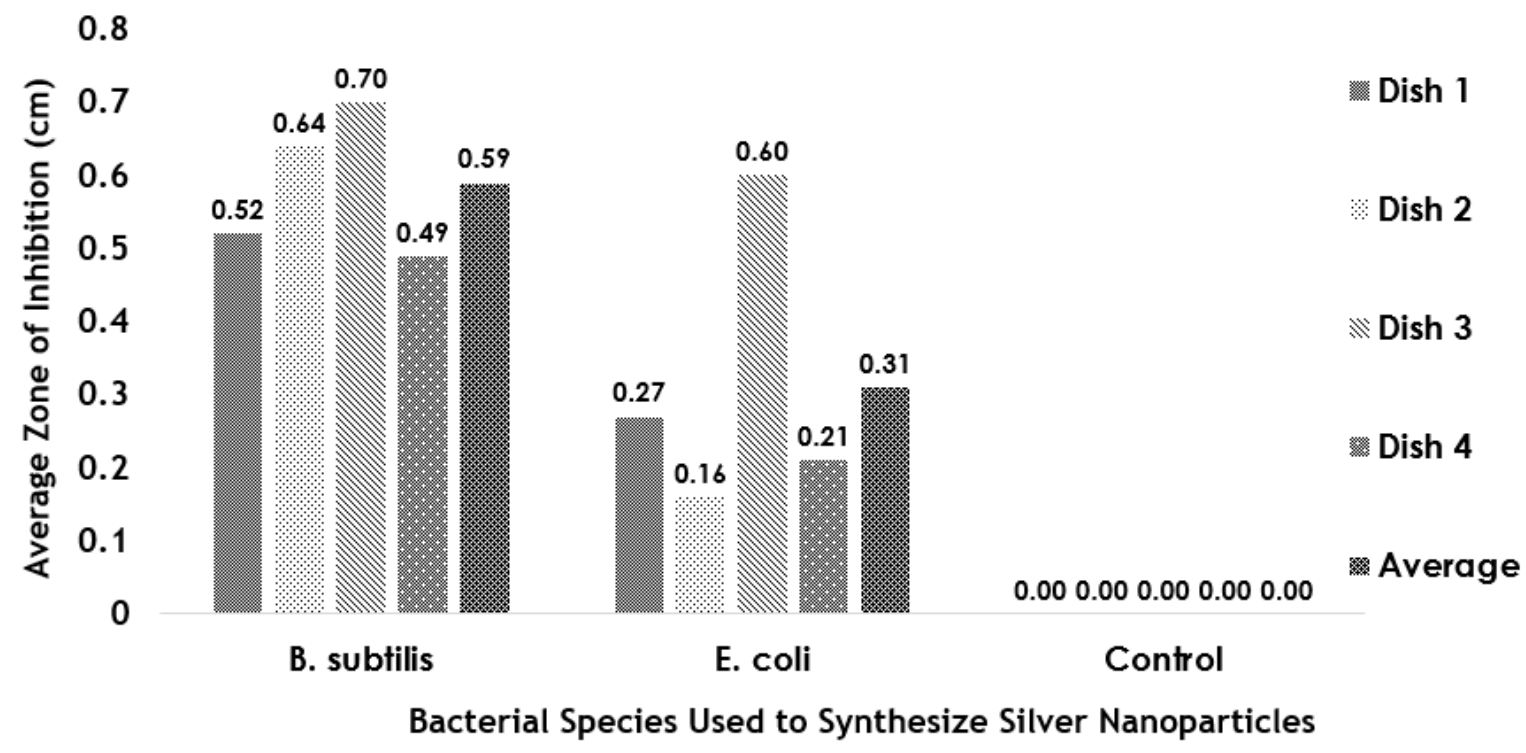

Figure 2. Results from agar well diffusion assay displayed graphically as measure of average zone of inhibition in $\mathrm{cm}$.

\section{Statistics}

Two layers of statistical analysis were done. The first one involved the analysis of the standard scores, the fractional number of standard deviations from the mean for each data point. Once again, the data from each of the four trials in every Petri Dish were averaged prior to computation of the standard scores (Table 2).

Table 2. Standard Score Results for Each of the Petri Dishes

\begin{tabular}{|c|c|c|c|c|c|}
\hline \multicolumn{7}{|c|}{ Standard Scores (Fractional Number of Standard Deviations from Mean) } \\
\hline & Dish 1 & Dish 2 & Dish 3 & Dish 4 & Average \\
\hline Control & 0 & 0 & 0 & 0 & 0 \\
\hline B. subtilis & -0.32 & 0.24 & 0.53 & -0.46 & 0 \\
\hline E. coli & 0 & 0 & 0 & 0 & 0 \\
\hline
\end{tabular}

The second layer of statistical analysis involved the calculation of the standard deviation, variance, and a Student's ttest. The standard deviation was calculated to be 0.308 and the variance 0.095 , both of which suggest that the data was concentrated and not many outlier data points affected the results. Additionally, a Student's t-test was performed to ensure the statistical significance of the results and the resulting value of 0.005 ensured that the trends seen in the data were because of internal factors rather than external ones. Overall, the statistical analyses conducted ensured that the results of the experiment were accurate and statistically significant.

\section{Discussion}

The results of the experiment clearly suggested that the silver nanoparticles created by B. subtilis were more effective than the ones produced by $E$. coli. This is proven by the fact that when comparing the average zones of inhibition for 
the different bacteria after 20 trials, it was found that the B. subtilis nanoparticles had on average a zone of inhibition that was $0.28 \mathrm{~cm}$ larger (Figure $2 \&$ Table 1). These results supported the idea that $B$. subtilis would be the preferred bacterial species for the production of silver nanoparticles in aerobic conditions because of how the assimilatory nitrate reduction process it uses was more effective in aerobic conditions and the nanoparticles were more effective. This is critical to research in the field which should increasingly focus on bacteria which utilize assimilatory nitrate reduction as they will be more effective in an aerobic industrial production facility. Standard deviation was calculated to be 0.308 and the variance 0.095 , both of which suggest that the data was concentrated and not many outlier data points affected the results. Additionally, a one-tailed Student's t-test found a resulting value of 0.005 which ensured that the trends seen in the data were because of internal factors rather than external ones. Overall, the statistical analyses conducted ensured that the results of the experiment were accurate and statistically significant.

The applications of this research and silver nanoparticles in general are innumerable. Currently silver nanoparticles offer the most effective method of treating antibiotic resistant bacterial growth. In particular, the effectiveness of silver nanoparticles in penetrating biofilm growth due to their miniscule nature is a core reason for their efficacy. The idea of treating antibiotic infections with silver nanoparticles has already taken shape in clinical trials with Cystic Fibrosis patients with antibiotic resistant infections where researchers are using a mist much like the flu mist to reach the airways of patients. However, the applications of silver nanoparticles also include the treatment of burn wounds which have some of the highest rates of infection. By applying silver nanoparticle gels in conjunction with other medicinal treatments, silver nanoparticles could prove pivotal in treating these wounds to prevent more serious infections from developing. Additionally, silver nanoparticles in conjunction with cellulose nanofibrous membranes can be used to filter water in areas where safe drinking water is not available for all due to a lack of infrastructure. The antibacterial properties of the silver nanoparticles in conjunction with the charge they have would allow them to not only attract particles, but also to kill any potentially dangerous microorganisms in the water.

\section{Conclusion}

To conclude, it is crucial to examine future areas of research. There are three potential future areas of research. The first primary area of research will focus on the potential of bacteria to develop resistance to silver nanoparticles. Currently, several studies reported by Salas Orozco, et al. have found no development of resistance to silver nanoparticles among a wide-ranging group of multi-drug resistant pathogens. However, isolated studies including Panacek, Kvitek, and Smekalova et al. have found repeated exposure of bacteria to silver nanoparticles can lead to the development of resistance in bacteria via the production of proteins that trigger the aggregation of the nanoparticles which limits their antibacterial activity. The second area of research will focus on the cytotoxic effects of silver nanoparticles and identifying a safe dosage for medical applications. Numerous studies have already been conducted concerning the cytotoxicity of silver nanoparticles on human cells including the one conducted by Liu, Fang et al. that highlights the toxicity and harmful effects of the nanoparticles on mitochondrial activity in neural stem cells. As the number of studies highlighting these potential effects increase, medical practitioners must determine a safe dosage and methods to limit the cytotoxic effects in order to bring silver nanoparticles into mainstream medicine. The final primary area of research to be conducted will center around improving the activity/effectiveness of green synthesized silver nanoparticles and comparing it to the activity of other antibacterial mechanisms including antibiotics. This could involve potential works into the synergistic antibacterial effects of antibiotics and nanoparticles.

\section{Limitations}

There were some limitations to the study that the author believed were important to highlight. First, due to safety restrictions, the study could only involve the bacterial species of $P$. fluorescens, which is a member of the same genus as $P$. aeruginosa. While the substitute bacteria ( $P$. fluorescens) may have very similar properties to $P$. aeruginosa, it 
is not exactly the same which means that further work must be conducted specifically on $P$. aeruginosa to validate the results and extend conclusions towards multi-drug resistant bacteria. Next, the durations during which the bacteria were allowed to grow were altered by 1-2 hours due to the constrictions placed on the researcher by the school scheduling. This should not have had any major impact on results. The final limitation on the research was how not all of the bacterial solution requiring centrifugation could be centrifuged at once due to the limitations of the equipment in the lab. Instead, the solution had to centrifuged in batches. Once again, this should not have impacted the results of the study significantly.

\section{Acknowledgements}

The researcher would like to thank everyone that helped with the study and providing the necessary equipment at Plano Senior High School. The researcher would like to thank his biology teacher, and LASER sponsor for their daily advice and for providing the lab materials/space required for the study. The study could not have been conducted without their help and guidance.

\section{References}

Ahmad, S., Munir, S., Zeb, N., Ullah, A., Khan, B., Ali, J., Bilal, M., Omer, M., Alamzeb, M., Salman, S. M., \& Ali, S. (2019). Green nanotechnology: a review on green synthesis of silver nanoparticles - an ecofriendly approach. International journal of nanomedicine, 14, 5087-5107. https://doi.org/10.2147/IJN.S200254

Bedlovičová, Z., \& Salayová, A. (2018). Green-Synthesized Silver Nanoparticles and Their Potential for Antibacterial Applications. Bacterial Pathogenesis and Antibacterial Control. https://doi.org/10.5772/intechopen.72138

Iravani, S., Korbekandi, H., Mirmohammadi, S. V., \& Zolfaghari, B. (2014). Synthesis of silver nanoparticles: chemical, physical and biological methods. Research in pharmaceutical sciences, 9(6), 385-406.

Liu, F., Mahmood, M., Xu, Y., Watanabe, F., Biris, A. S., Hansen, D. K., Inselman, A., Casciano, D., Patterson, T. A., Paule, M. G., Slikker, W., \& Wang, C. (2015). Effects of silver nanoparticles on human and rat embryonic neural stem cells. Frontiers in Neuroscience.

https://doi.org/10.3389/fnins.2015.00115

Saifuddin, N., Wong, C. W., \& Yasumira, A. A. N. (2009). Rapid Biosynthesis of Silver Nanoparticles Using Culture Supernatant of Bacteria with Microwave Irradiation. E-Journal of Chemistry, 6(1), 61-70. https://doi.org/10.1155/2009/734264

Salomoni, R., Léo, P., Montemor, A. F., Rinaldi, B. G., \& Rodrigues, M. (2017). Antibacterial effect of silver nanoparticles in Pseudomonas aeruginosa. Nanotechnology, science and applications, 10, 115-121.

https://doi.org/10.2147/NSA.S133415

Salas-Orozco, M., Niño-Martínez, N., Martínez-Castañón, G.-A., Méndez, F. T., Jasso, M. E. C., \& Ruiz, F. (2019). Mechanisms of Resistance to Silver Nanoparticles in Endodontic Bacteria: A Literature Review. Journal of Nanomaterials, 2019. https://doi.org/10.1155/2019/7630316 
Shehzad, A., Qureshi, M., Jabeen, S., Ahmad, R., Alabdalall, A. H., Aljafary, M. A., \& Al-Suhaimi, E. (2018). Synthesis, characterization and antibacterial activity of silver nanoparticles using Rhazya stricta. PeerJ, 6 , e6086.

https://doi.org/10.7717/peerj.6086

Siddiqi, K. S., Husen, A., \& Rao, R. A. K. (2018). A review on biosynthesis of silver nanoparticles and their biocidal properties. Journal of Nanobiotechnology, 16(1). https://doi.org/10.1186/s12951-018-0334-5

Panáček, A., Kvítek, L., Smékalová, M., Večeřová, R., Kolář, M., Röderová, M., Dyčka, F., Šebela, M., Prucek, R., Tomanec, O., \& Zbořil, R. (2017). Bacterial resistance to silver nanoparticles and how to overcome it. Nature Nanotechnology, 13(1), 65-71. https://doi.org/10.1038/s41565-017-0013-y

Qing, Y., Cheng, L., Li, R., Liu, G., Zhang, Y., Tang, X., Wang, J., Liu, H., \& Qin, Y. (2018). Potential antibacterial mechanism of silver nanoparticles and the optimization of orthopedic implants by advanced modification technologies. International journal of nanomedicine, 13, 3311-3327.

https://doi.org/10.2147/IJN.S165125

Zhang, X. F., Liu, Z. G., Shen, W., \& Gurunathan, S. (2016). Silver Nanoparticles: Synthesis, Characterization, Properties, Applications, and Therapeutic Approaches. International journal of molecular sciences, 17(9), 1534. https://doi.org/10.3390/ijms17091534 